S. SASAO AND J.-S. CHA

KODAI MATH. $J$.

13 (1990), 231-240

\title{
ON SELF-HOMOTOPY EQUIVALENCES OF COVERING SPACES
}

\author{
By Seiya Sasao and Cha Joon-Sim
}

\section{$\S 0$. Introduction.}

Let $X$ be a $G$-space and we denote by $K X$ the space of continuous maps from $K$ into $X$ endowed with Compact-Open topology. Since a $G$-action on the space $K X$ is naturally induced we may regard the path-connected components of $K X, \pi_{0}(K X)$, as a $G$-set. Then we are interested in the isotropy subgroup $G\langle f\rangle$ at the homotopy class of a map $f: K \rightarrow X$. This relates to othor problems as follows:

(1) When $K$ is considered as a trivial $G$-space a map $f$ is equivariant up to homotopy if and only if $G\langle f\rangle=G$, namely $f$ is a fixed element.

(2) Since the $G$-action on $X$ is given by a continuous map $\Phi: G \rightarrow X X$ we have an induced homomorphism $\Phi_{*}: G \rightarrow \varepsilon(X)$, the group of homotopy classes of self-homotopy equivalences. Then $G\left\langle 1_{x}\right\rangle$ is just the kernel of $\Phi$.

(3) Of course the determination of $G\langle f\rangle$ for all $f$ gives us some informations on the structure of the set $\pi_{0}(K X)$.

As the first step of our program, in this paper we are mainly concerned with the case of covering spaces and their deck transformation groups. Then there are a few points of view about categories:

(1) The category of 0 -connected $C W$-complexes and maps of base-point free.

(2) The sub-category of fibre-preserving maps.

(3) The sub-category of equivariant maps.

We work in these categories to investigate the kernel of $\Phi_{*}: G \rightarrow \varepsilon(X)$. As results, we obtain some exact sequences for a regular covering $p: X \rightarrow Y$ with its deck transformation group $G$ as follows:

(1) $\{1\} \rightarrow \Gamma(X, Y ; p) / \Gamma(X) \rightarrow G \rightarrow \varepsilon(X)$

(2) $\{1\} \rightarrow \Gamma(Y) / \Gamma_{F}(X) \rightarrow G \rightarrow \varepsilon_{F}(X) \rightarrow \varepsilon_{L}(Y) \rightarrow\{1\}$

(3) $\{3\} \rightarrow \Gamma(Y) / \Gamma_{G}(X) \rightarrow Z[G] \rightarrow \varepsilon_{G}(X)$

(see the context about notations)

For example, let $p: R^{n} \rightarrow Y$ be a universal covering and $G$ be the groun $\pi_{1}(Y, *)$, then we have an exact sequence

Received October 13, 1989; Revised December 7, 1989, 


$$
\{1\} \longrightarrow Z[G] \longrightarrow G \longrightarrow \varepsilon_{F}\left(R^{n}\right) \longrightarrow \text { Aut. } G \longrightarrow\{1\} \text {. }
$$

\section{$\S 1 . \pi_{1}(X, *)$-action on the set $[K, X]_{0}$}

First we recall a notion from the homotopy theory [5], [8]. Let us denote by $[K, X]_{0}$ the set of homotopy classes of base-point preserving maps. Then, for every loop $\omega$ of $X$ at $*$ and a base-point preserving map $f: K \rightarrow X$, there exists a map $\phi: I \times K \rightarrow X$ which is an extension of the map

$$
\omega \cup f: I \times * \cup 0 \times K \longrightarrow X \text {. }
$$

Since the homotopy class of the restriction of $\phi$ on $1 \times K$ depends on only homotopy classes of $\omega$ and $f$ this defines an action $\omega^{\#}$ of $\pi_{1}(X, *)$ on the set $[K, X]_{0}$. On the other hand this action can be reformulated as follows:

Let $p: K X \rightarrow X$ be the fibring defined by $p(f)=f(*)$. Clearly the fibre over $*$ is the space of base-point preserving maps, $\{K, X\}_{0}$, and we have the part of the homotopy exact sequence

$$
S_{1}: \pi_{1}(K X, f) \underset{p_{*}}{\longrightarrow} \pi_{1}(X, *) \underset{\partial_{f}}{\longrightarrow} \pi_{0}\left(\{K, X\}_{0}\right) \longrightarrow \pi_{0}(K X, f) \longrightarrow\{f\} .
$$

Then it holds $\partial_{f}(\omega)=\omega^{\#}(f)$.

Since it is clear that a loop $\omega$ is contained in the image of $p_{*}$ if and only if there exists a map: $S^{1} \times K \rightarrow X$ of type $(\omega, f)$ we have

LEMMA 1.1. $\omega^{\sharp}(f)=f$ holds if and only if there exists $a$ map: $S^{1} \times K \rightarrow X$ of type $(\omega, f)$,

Here we note a property of the $\pi_{1}$-action above which easily follows from the definition.

Lemma 1.2. For two maps $f:(X, *) \rightarrow(Y, *)$ and $g:(Y, *) \rightarrow(Z, *)$ we have

$$
\tau^{\sharp}(g f)=\tau^{\#}(g) f \quad \text { ond } \quad g_{*}(\omega)^{\#}(g f)=g\left(\omega^{\#}(f)\right)
$$

where $\tau$ and $\omega$ are elements of $\pi_{1}(Y, *)$ and $\pi_{1}(Z, *)$ respectively.

For example we prove

Proposition 1.3. If $f:(Y, *) \rightarrow(X, *)$ is a homotopy equivalence then $\omega^{\#}(f)$ is also a homotopy equivalence for any $\omega$ of $\pi_{1}(X, *)$.

Proof. First it is shown that $\omega^{\sharp}\left(1_{X}\right)$ is a homotopy equivalence because we have

$$
\left(\omega^{-1}\right)^{\#}\left(1_{X}\right) \omega^{\#}\left(1_{X}\right)=\left(\omega^{-1}\right)^{\#}\left(1_{X} \omega^{\#}\left(1_{X}\right)\right)=\left(\omega^{-1}\right)^{\#} \omega^{\#}\left(1_{X}\right)=1_{X}
$$


and similarly $\omega^{\#}\left(1_{X}\right)\left(\omega^{-1}\right)^{\#}\left(1_{X}\right)=1_{X}$. Secondly, let $g$ be a homotopy inverse of $f$. Then we have

$$
g\left\{\omega^{\#}(f)\right\}=g_{*}(\omega)^{\#}(g f)=\left\{g_{*}(\omega)\right\}^{\#}\left(1_{X}\right) \quad \text { and } \quad \omega^{\#}(f) g=\omega^{\#}(f g)=\omega^{\#}\left(1_{X}\right) .
$$

and Thus it follows from the first case that $\omega^{\#}(f)$ has a right and left inverse respectively and hence $\omega^{\sharp}(f)$ is a homotopy equivalence.

As an example we consider the case of $K=X$ and $f=1_{X}$ in the based category. Then the exact sequence $S_{1}$ is turned into the sequence

$$
S_{2}: \pi_{1}\left(X X, 1_{X}\right) \longrightarrow \pi_{1}(X, *) \longrightarrow\left([X, X]_{0}, 1_{X}\right) \longrightarrow\left([X, X], 1_{X}\right) \longrightarrow\left\{1_{X}\right\} .
$$

Now we define a multiplication in the set $[X, X]$ by the composite of maps, which makes the set a semi-group with $1_{X}$ as unit. Since we have

$$
\partial(\omega \cdot \tau)=(\omega \tau)^{\#}\left(1_{X}\right)=\omega^{\#}\left(\tau^{\#}\left(1_{X}\right)\right)=\omega^{\#}\left(1_{X} \tau^{\#}\left(1_{X}\right)\right)=\omega^{\#}\left(1_{X}\right) \tau^{\#}\left(1_{X}\right)=(\partial \omega)(\partial \tau)
$$

the following lemma holds.

LEMMA 1.4. $\partial$ is homomorphic in the sequence $S_{2}$.

Since, for a class $h$ of a homotopy equivalence: $(X, *) \rightarrow(X, *), \omega^{\#}(h)$ is also a homotopy equivalence the sequence $S_{2} 1$ s transformed by Proposition 1.3 into an exact sequence in the category of groups and homomorphisms

$$
S_{3}: \pi_{1}\left(X X, 1_{X}\right) \longrightarrow \pi_{1}(X, *) \longrightarrow \varepsilon_{0}(X) \longrightarrow \varepsilon(X) \longrightarrow\left\{1_{X}\right\}
$$

where $\varepsilon_{0}(X)$ denotes the group consisting of invertible elements of $[X, X]_{0}$.

Now we define a (normal) subgroup of $\pi_{1}(X, *)$ by

$$
\Gamma(X)=\left\{\omega \mid \text { there exists a map: } S^{1} \times X \rightarrow X \text { of type }\left(\omega, 1_{X}\right)\right\} .
$$

LEMMA 1.5. $\Gamma(X)$ is contained in the centre of $\pi_{1}(X, *)$ (see page 843 of [2]).

Proof. For $\tau$ of $\pi_{1}(X, *)$ and $\omega$ of $\Gamma(X)$ a map: $S^{1} \times S^{1} \rightarrow X$ of type $(\omega, \tau)$ is given by the composite $S^{1} \times S^{1} \rightarrow S^{1} \times X \rightarrow X$. Hence Whitehead product $[\tau, \omega]$ is trivial, i. e. $\tau \omega=\omega \tau$.

Since we know $\partial^{-1}\left(1_{X}\right)=\Gamma(X)$ from lemma 1.2 we have

THEOREM 1.6. There exists an exact sequence

$$
\{1\} \longrightarrow \pi_{1}(X, *) / \Gamma(X) \longrightarrow \varepsilon_{0}(X) \longrightarrow \varepsilon(X) \longrightarrow\{1\} .
$$

Thus Theorem 1.6 and lemma 1.5 give

COROLLARY 1.7. If the centre of $\pi_{1}(X, *)$ is trivial we have an exact sequence 


$$
\{1\} \longrightarrow \pi_{1}(X, *) \longrightarrow \varepsilon_{0}(X) \longrightarrow \varepsilon(X) \longrightarrow\{1\} \text {. }
$$

As another example, let $P_{m}$ be the pseud-projective plane $S^{1} \cup_{m} e^{2}$. Since it follows from a cohomological considration that $\Gamma\left(P_{m}\right)$ is trivial we have a short exact sequence $([1],[4])$

$$
\{1\} \longrightarrow Z_{m} \longrightarrow \varepsilon_{0}(X) \longrightarrow \varepsilon(X) \longrightarrow\{1\} .
$$

\section{§ 2. Regular Covering spaces.}

In this section our argumrnt is related to the paper [7]. Let $p: X \rightarrow Y$ be a regular covering, i.e. $p_{*}\left(\pi_{1}(X, *)\right)$ is a normal subgroup of $\pi_{1}(Y, *)$ and Let $G$ be the deck transformation group of $p$. Then for any locally compact and locally path-connected Hausdorff space $K$ we have

LEMMA 2.1. The naturally induces map $p^{K}: K X \rightarrow K Y$ is a fibre space whose fibre over $p f$ is $G f$ for any map $f: K \rightarrow X$ where the action of $G$ on $K X$ is given by $G \times K X \rightarrow K X:(g, h) \rightarrow g h$.

Consider a part of the homotopy exact sequence of $p^{K}$

$$
S_{4}: \pi_{1}(K X, f) \longrightarrow \pi_{1}(K Y, p f) \longrightarrow(G, *) \longrightarrow \pi_{0}(K X, f) \longrightarrow \pi_{0}(K Y, p f)
$$

for a map $f: K \rightarrow X$ where we identify $G f$ with $G$. Then a standard argument gives

LEMMA 2.2. The boundary $\pi_{1}(K Y, p f) \rightarrow(G, *)$ is homomorphic, and the correspondence $(G, *) \rightarrow \pi_{0}(K X, f)$ is naturally induced by the action of $G$ on $K X$.

Now consider the following commutative diagram obtained from fibrings

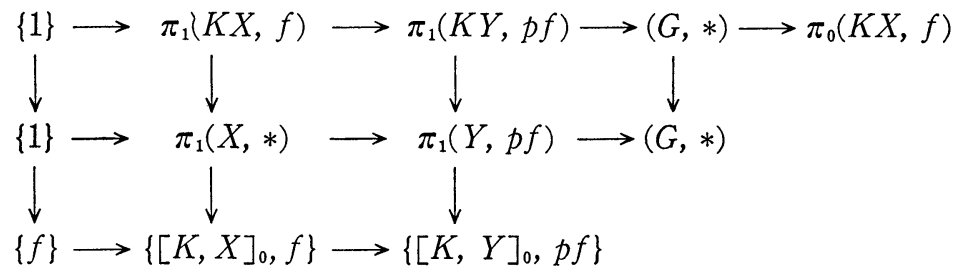

and use the following notation for a map $h:(A, *) \rightarrow(B, *) \Gamma(A, B: h)=$ $\left\{\omega \mid \omega \in \pi_{1}(B, *)\right.$ and there exists a map $S^{1} \times A \rightarrow B$ of type $\left.(\omega, h)\right\}$.

Then, using lemma 2.2 , we can easily obtain

PROPOSITION 2.3. Let $p: X \rightarrow Y$ be a regular covering whose deck transformation group is $G$. Then $G\langle f\rangle$ is isomorphic to $\Gamma(K, Y: p f) / \Gamma(K, X: f)$

For a regular covering $p: X \rightarrow Y$ we have as applications of PROP. 2.3 
COLLORARY 2.4. There exists an exact sequence

$\{1\} \longrightarrow \Gamma(K, Y: p f) / \Gamma(K, X: f) \longrightarrow \pi^{1}(Y, *) \longrightarrow \pi_{1}(X, *) \longrightarrow\{[K, X], f\}$

Since $\Gamma\left(X, X: 1_{X}\right)=\Gamma(X)$ (see $\left.\S 1\right)$, as a special case, we have

COLLORARY 2.5. There exists an exact sequence

$$
\{1\} \longrightarrow \Gamma(X, Y: p) / \Gamma(X) \longrightarrow G \longrightarrow \varepsilon(X) \longrightarrow\{[X, Y], p\} .
$$

As another application we have

COLlORARY 2.6. A map $f: S^{n} \rightarrow X(n \geqq 2)$ is G-equivariant up to homotopy if and only if all Whitehead products $\left[\pi_{1}(Y, *), p f\right]$ vanish.

Let $p: X \rightarrow Y$ be a covering space which is not necessarly regular. Then, noting $p(k)^{-1}(p f)=G f$, the sequence $S_{4}$ turns out the sequence,

$$
\pi_{1}(K X, f) \longrightarrow \pi_{1}(K Y, p f) \longrightarrow\left(G f, e_{0} f\right) \longrightarrow \pi_{0}(K X, f) \longrightarrow \pi_{0}(K Y, p f)
$$

which relates to other sequences as follows:

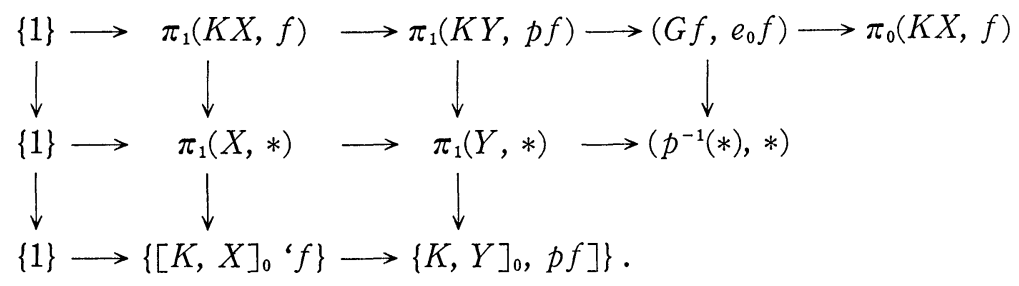

Let denote by $N\left(\pi_{1}(X, *)\right)$ the normalizer of $\pi_{1}(X, *)$ in $\pi_{1}(Y, *)$ and $\Gamma(K, Y: f)$ be the intersection $\Gamma(K, Y: p f) \cap N\left(\pi_{1}(X, *)\right)$. Since $G$ is isomorphic to $N\left(\pi_{1}(X, *)\right) / \pi_{1}(X, *)$, using the above diagram and argument similar to the case of regular coverings we can obtain the following

Proposition 2.7. Let $p: X \rightarrow Y$ be a covering space with its deck transformation group $G$ and $f$ be a map $(X, *) \rightarrow(Y, *)$. Then $G\langle f\rangle$ is isomorphic to $\Gamma(K, Y: p f) / \Gamma(K, X: f)$.

\section{$\S 3$. Orbits (fibre)-preserving maps.}

For a regular covering $p: X \rightarrow Y$ we denote by $F(X)$ the space of orbits preserving maps, i.e. $f: X \rightarrow X$ satisfying $p f(g x)=p f(x)$ for all $x, g$. Then we have the pull-buck diagram of fibrings derived from the covering 


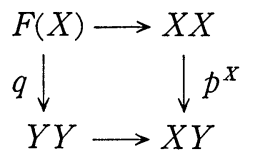

where $Y Y \rightarrow X Y$ is given by composite $X \rightarrow Y \rightarrow Y$. Since we may consider $G$ as the fibre $q^{-1}\left(1_{Y}\right)$ we have the commutative diagram of a part of homotopy exact sequences ;

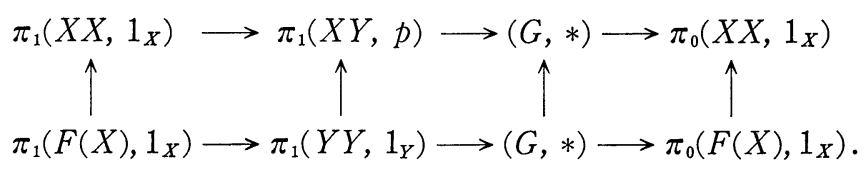

Then, as the same as the case of the upper sequqnce, we can know that the lower sequence is an exact sequence of semi-groups and homomorphisms, We denote by $\varepsilon_{F}(X)$ the group consisting of invertible elements of $\pi_{0}\left(F(X), 1_{X}\right)$, and obtain the following diagram from the above one

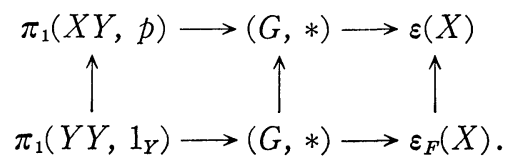

We define subgroup of $\pi_{1}(X, *)$ by

$\Gamma_{F}(X)=\left\{\tau \mid\right.$ there exist an orbits-preserving map $S^{1} \times X \rightarrow X$ of type $\left.\left(\tau, 1_{X}\right)\right\}$

Proposition 3.1. The image of the boundary $\pi_{1}\left(Y Y, 1_{Y}\right) \rightarrow(G, *)$ in the lower sequence is isomorphic to $\Gamma(Y) / \Gamma_{F}(X)$.

Proof. The proof follows from the argument analogus to PROP. 2.3 and the diagram,

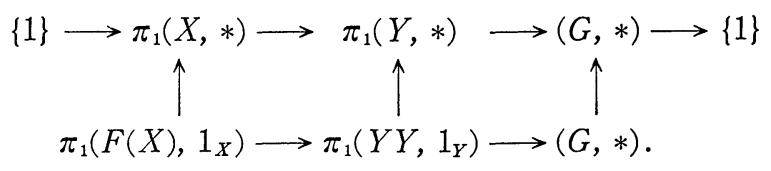

COROllaRy 3.2. The image: $\pi_{1}\left(Y Y, 1_{Y}\right) \rightarrow(G, *)$ is contained in the center of $G$.

Proof. For $\omega$ of $\pi_{1}(Y, *)$, assume that there exists a map: $S^{1} \times Y \rightarrow Y$ of type $\left(\omega, 1_{Y}\right)$. Then for any $\sigma$ of $\pi_{1}(Y, *)$, a map: $S^{1} \times S^{1} \rightarrow Y$ of type $(\omega, \sigma)$ is given by composite $S^{1} \times S^{1} \rightarrow S^{1} \times Y \rightarrow Y$. Hence Whitehead procuct $[\omega, \sigma]$ is trivial, i.e. $\omega \sigma=\sigma \omega$. Since $\pi_{1}(Y, *) \rightarrow G$ is onto the proof is completed.

Example 3.3. Let $X \rightarrow Y$ be the universal covering. If the centre of $\pi_{1}(Y, *)=G$ is trivial (e. g. $G$ : simple) then $\varepsilon_{F}(X)$ contains $\pi_{1}(Y, *)$ as a subgroup. 
Next we consider the homomorphism $q .: \pi_{0}\left(F(X), 1_{X}\right) \rightarrow \pi_{0}\left(Y Y, 1_{Y}\right)$ induced by the projection $q$. Let us denote by $L(Y)$ the image of $q$. and by $\varepsilon_{L}(Y)$ the group consisting of invertible elements of $L(Y)$.

LEMma 3.4. The homomorphism $\varepsilon_{F}(X) \rightarrow \varepsilon_{L}(Y)$ is surjective.

Proof. Consider the commutative diagarm



and let $\tilde{g}$ be a homotopy inverse of $\tilde{f}$. Since we may assume teat $\tilde{f}$ and $\tilde{g}$ are base-point preserving maps the following equalities hold for a loop $\omega$ at $*$

$$
\omega^{-1} p_{*} \pi_{1}(X, *) \omega=\omega^{\#} p_{*} \pi_{1}(X, *)=\tilde{g}_{*} f_{*} p_{*} \pi_{1}(X, *)=\tilde{g}_{*} p_{*} \pi_{1}(X, *) .
$$

Thus we have $\tilde{g}_{*} p_{*} \pi_{1}(X, *)=p_{*} \pi_{1}(X, *)$ from the normality of $p_{*} \pi_{1}(X, *)$ in $\pi_{1}(Y, *)$, and this means that $\check{g}$ is liftable, i. e. there exists a map $g: X \rightarrow X$ such that $\tilde{g} p=p g$. Then, from $q_{*}(f g)=1_{Y}$ and exactness of the sequence, we can know that $f$ is invertible in $\pi_{0}\left(F(X), 1_{X}\right)$

Now combining PROP. 3.1 with lemma 3.4 we have

THEOREM 3.5. For a regular covering $p: X \rightarrow Y$ there exists an exact sequence

$$
\{1\} \longrightarrow \Gamma(Y) / \Gamma_{F}(X) \longrightarrow \varepsilon_{F}(X) \longrightarrow \varepsilon_{L}(Y) \longrightarrow\{1\} \text {. }
$$

Example 3.6. For the universal covering $p: X \rightarrow Y$ we have an exact sequence: $\{1\} \rightarrow \Gamma(Y) \rightarrow \pi_{1}(Y, *) \rightarrow \varepsilon_{F}(X) \rightarrow \varepsilon_{L}(Y) \rightarrow\{1\}$.

Example 3.7. Let $p: \boldsymbol{R}^{n} \rightarrow Y$ be the universal covering and let $G$ be $\pi_{1}(Y, *)$. Since it can be easily shown that the center of $G, Z[G]$, is isomorphic to $\Gamma(Y)$ and that $\varepsilon_{L}(Y)$ is also isomorphic to AUT. $G$ we have an exact sequence

$$
\{1\} \longrightarrow Z[G] \longrightarrow G \longrightarrow \varepsilon_{F}\left(\boldsymbol{R}^{n}\right) \longrightarrow \text { AUT. } G \longrightarrow\{1\} .
$$

Especially if $G$ is abelian we have an isomorphism $\varepsilon_{F}\left(\boldsymbol{R}^{n}\right) \simeq$ AUT. $G$.

\section{$\S 4$. Equivariant maps.}

Since equivariant maps are a kind of typical orbits-preserving maps we shall study the space of those maps, which is denoted by $E_{q}(X)$.

First by James's result (Theorem (2.1) of [2]) we have a commutative diagram of fibrings 


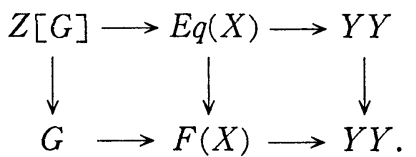

Remark. This diagram contains an easy proof of Corollary 3.2.

Define a subgroup of $\pi_{1}(X, *)$ by

$\Gamma_{G}(X)=\left\{\tau \mid\right.$ there exists an equivariant map: $S^{1} \times X \rightarrow X$ of type $\left.\left(\tau, 1_{X}\right)\right\}$

Then using the diagram above and argument analogus to the preceeding section we have

Proposition 4.1. There exists an exact sequence

$$
\{1\} \longrightarrow \Gamma(Y) / \Gamma_{G}(X) \longrightarrow Z[G] \longrightarrow \varepsilon(X) .
$$

where $\varepsilon_{G}(X)$ denotes the subgroup of $\pi_{0}(E q(X))$ consisting of invertible elements, i.e. homotopy classes of self-homotopy equivalences in the equivariant category.

Now we prove

Proposition 4.2. The homomorphism: $\varepsilon_{G}(X) \rightarrow \boldsymbol{\varepsilon}_{F}(X)$ is injective.

For the proof we note

LEMMA 4.4. Let $X_{k}$ be a properly discontinuous free $G$-space $(k=1,2)$ and $f$ be a map $X_{1} \rightarrow X_{2}$ such that $p_{2} f(g x)=p_{2} f(x)$ where $p_{k}: X_{k} \rightarrow Y_{k}$ is the projection onto the space of orbits. Clearly $f$ defines a correspondence $\rho_{f}: G \times X_{1} \rightarrow G$ by $f(g x)=\rho_{f}(g, x) f(x)$. Then $\rho_{f}$ is continuous.

Proof of Proposition 4.2. Let $f$ be an equivariant map: $X \rightarrow X$ and $H$ be a homotopy between $f$ and $1_{X}$ in the space $F(X)$, i. e. $H: I \times X \rightarrow X$ satisfies

$$
H(0, x)=f(x), \quad H(1, x)=x \quad \text { and } \quad p H(l, g x)=p H(t, x) .
$$

Applying lemma 4.3 to the case of $X_{1}=I \times X, X_{2}=X, H$ defines a continuous map $\rho: I \times X \times G \rightarrow G$ satisfying

$$
H(g(t, x))=H(t, g x)=\rho(t, x, g) H(t, x) .
$$

Since $G$ is discreate we know $\rho(t, x, g)=\rho(0, *, g)$ for all $t$ and $x$. On the other hand, from equalities:

$$
\rho(0, *, g) f(*)=\rho(0, *, g), \quad H(0, *)=H(0, g *)=f(g *)=g f(*)
$$

it follows $\rho(0, *, g)=g$. Thus we have $H(t, g x)=g H(t, x)$, which means $H$ is an equivariant homotopy between $f$ and $1_{X}$. This completes the proof. 
Now let $f: X \rightarrow X$ be a map satisfying $p f(x)=p(f(g x))$, i. e. $f \in F(X)$. By lemma 4.3 there exists a continuous map $\rho_{f}: G \times X \rightarrow G$ with $f(g x)=\rho_{f}(g, x) f(x)$. Again, since $G$ is discrete this turns out a correspoudence

$$
\rho_{f}^{*}: G \longrightarrow G \quad \text { with } f(g x)=\rho_{f}^{*}(g) f(x) .
$$

LEMMA 4.4. $\rho_{f}{ }^{*}$ is an endmorphism of $G$, and $\rho_{f_{1}}{ }^{*}=\rho_{f_{2}}{ }^{*}$ if $f_{1}$ is homotopic to $f_{2}$ in the space $F(X)$.

Proof. The first follows from

$$
\rho_{f}^{*}\left(g_{1}, g_{2}\right) f(*)=f\left(g_{1} g_{2} *\right)=\rho_{f} *\left(g_{1}\right) f\left(g_{2} *\right)=\rho_{f} *\left(g_{1}\right) \rho_{f} *\left(g_{2}\right) f(*)
$$

and the second is easily shown by an argument anaolgus to the proof of Proposition 4.2

Thus $\rho_{f}^{*}$ gives another correspondence

$$
[\rho]: \pi_{0}(F(X)) \longrightarrow \text { End. } G
$$

defined by $[\rho](f)=\rho_{f} *$.

LEMMA 4.5. [ $\rho]$ is homomorphic, and hence this induces a homomorphasm: $\varepsilon_{F}(X) \rightarrow$ Aut. $G$, whose kernel is isomorphic to $\varepsilon_{G}(X)$.

Proof. Let $f_{k}: X \rightarrow X(k=1,2)$ be maps in the space $F(X)$. Then equalities

$$
\rho_{f_{1} f_{2}} *(g)\left(f_{1} f_{2}\right)(*)=\left(f_{1} f_{2}\right)(g *)=f_{1}\left(f_{2}(g *)\right)=f_{1}\left(\rho_{f_{2}} *(g) f_{2}(*)=\rho_{f_{1}} *\left(\rho_{f_{2}} *(g)\right) f_{1} f_{2}(*)\right)
$$

gives the proof.

Thus, from lemma 4.5 and Proposition 4.2 , we obtain

Proposition 4.6. There exists an exact sequence:

$$
\{1\} \longrightarrow \varepsilon_{G}(X) \longrightarrow \varepsilon_{F}(X) \longrightarrow \text { Act. } G \text {. }
$$

In general it seems to be difficult to obtain some characterization of the image: $\varepsilon_{F}(X) \rightarrow$ Aut. $G$.

Remark. There is another interpretation of the homomorphism $[\rho]$ above, namely consider two covering spaces with base point as follows:

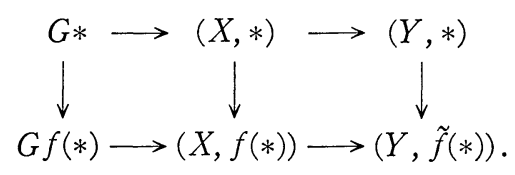

Then we have a commutative diagram: 




Example 4.7. If $\boldsymbol{R}^{n} \rightarrow Y$ is a regular covering and $\pi_{1}(Y, *)$ is abelian then $\boldsymbol{\varepsilon}_{G}\left(\boldsymbol{R}^{n}\right)$ is trivial $\left(G=\pi_{1}(Y, *)\right)$ (Example 3.1 of [6]).

\section{REFERENCES}

[1] H.J. Baues, The homotopy category of pseudo-projective planes. (preprint).

[2] D. H. Gottlieb, A certain subgroup of the fundamental group. Amer. J. of Math. 87 (1965), 840-856.

[3] I.M. James, The space of bundle maps. Topology 2 (1963), 45-59.

[4] J.W. RUTTER, Homotopy classification of maps between pseudo-projective planes. (preprint).

[5] E.H. Spanier, Algebraic Toplogy. 1966 (McGraw-Hill).

[6] K. Tsukiyama, Equivariant self equivalences of principale fibre bunbundles. Math. Proc. of Camb. Phil. Soc. 98 (1985), 87-92.

[7] K. Varadarajan, Generalized Gottlieb groups. J. Indian Math. Soc. 33 (1969), 141-164.

[8] G.W. WhiteheAd, Elements of Homotopy Theory. GTM. 61. 1978 (SpringerVerlag).

DEPT. OF MATH.

TOKYo Institute of TECHNOLOGY

OHOKayama Meguro-Ku TOKYo

JAPAN 